\title{
Rotation of the Nucleus, Gas Kinematics and Emission Pattern of Comet 8P/Tuttle: Preliminary Results from Optical Imaging of the CN Coma
}

\author{
W. Waniak · G. Borisov · M. Drahus · T. Bonev $\cdot$ K. Czart • \\ M. Küppers
}

Received: 29 December 2008/ Accepted: 28 May 2009/Published online: 18 June 2009

(C) The Author(s) 2009. This article is published with open access at Springerlink.com

\begin{abstract}
We present preliminary results of the narrow-band $\mathrm{CN}$ observations of comet 8P/Tuttle from early January 2008, realized as part of a project consisting of nearsimultaneous spectroscopic monitoring of $\mathrm{HCN}$ at millimeter-wavelengths and optical imaging of the coma. The mean-image subtraction method revealed low-contrast $\mathrm{CN}$ envelopes. Using the image cross-correlation technique we measured the projected velocity of these shells. For the sunward part we found it to be equal to $0.96 \pm 0.03 \mathrm{~km} \mathrm{~s}^{-1}$ on January 4 and $1.10 \pm 0.01 \mathrm{~km} \mathrm{~s}^{-1}$ on January 9 , whereas the anti-sunward part reached $0.73 \pm 0.05$ and $0.80 \pm 0.02 \mathrm{~km} \mathrm{~s}^{-1}$, respectively. The periodicity of gas emission was investigated using a repeatability of the shells, their kinematics, and an aperture photometry of the near nucleus region. We found a period of $5.70 \pm 0.07 \mathrm{~h}$ (along with multiples), consistent with previous findings by other authors. A toy Monte Carlo model was implemented to reproduce the time-series of the $\mathrm{CN}$ images. We show that emission of $\mathrm{HCN}$ into a relatively wide cone by a single active region on a rotating nucleus is the most probable scenario.
\end{abstract}

Electronic supplementary material The online version of this article (doi:10.1007/s11038-009-9326-7) contains supplementary material, which is available to authorized users.

W. Waniak $(\bowtie)$

Astronomical Observatory of the Jagiellonian University, ul. Orla 171, 30-244 Kraków, Poland e-mail: waniak@oa.uj.edu.pl; wwaniak@w.krakow.pl

G. Borisov · T. Bonev

Institute of Astronomy of the Bulgarian Academy of Sciences, 72 Tsarigradsko Chaussee Blvd., 1784

Sofia, Bulgaria

M. Drahus

Max-Planck-Institut für Sonnensystemforschung, Max-Planck-Str. 2, 37191 Katlenburg-Lindau,

Germany

K. Czart

Toruń Centre for Astronomy of the Nicolaus Copernicus University, ul. Gagarina 11, 87-100 Toruń, Poland

M. Küppers

European Space Astronomy Centre, Villanueva de la, Cañada, P.O. Box 78, 28691 Madrid, Spain 
Keywords Comets $\cdot$ Comet $8 \mathrm{P} / \mathrm{Tuttle} \cdot \mathrm{CN}$ coma $\cdot$ Gas emission $\cdot$ Nucleus rotation

\section{Introduction}

Comet 8P/Tuttle belongs to the Halley-type Comets which constitute, along with the longperiod and the dynamically-new comets, a global group of the Nearly Isotropic Comets (Levison 1996). Most probably they come from the Oort cloud but had been created in the zone of the giant planets. In spite of relatively frequent perihelion passages since its discovery in the middle of the nineteenth century (the orbital period is about 13.6 year), little was known about comet Tuttle to date (e.g. the previous perihelion passage in 1994 suffered from highly unfavourable geometric conditions). One of the main results from the previous studies was that the $\mathrm{CN}$ and $\mathrm{C}_{2}$ abundances are quite normal, but the comet is depleted in dust (A'Hearn et al. 1995). From snap-shot photometry of the nearly inactive nucleus we additionally learned that its effective radius is close to $7 \mathrm{~km}$ (Licandro et al. 2000; Snodgrass et al. 2008).

In contrast, the last perihelion passage of $8 \mathrm{P} /$ Tuttle, which took place at the turn of $2007 / 2008$, was the most favourable in the recent history of this object (the minimum geocentric distance of $0.25 \mathrm{AU}$ ) providing a unique opportunity to uncover its physical nature in detail. The comet was studied with imaging and spectroscopy across all wavelengths. A number of volatiles, such as $\mathrm{H}_{2} \mathrm{O}, \mathrm{H}_{2} \mathrm{~S}, \mathrm{CS}, \mathrm{HCN}, \mathrm{HNC}, \mathrm{CH}_{3} \mathrm{CN}, \mathrm{CH}_{4}, \mathrm{C}_{2} \mathrm{H}_{2}$, $\mathrm{C}_{2} \mathrm{H}_{6}, \mathrm{CO}, \mathrm{H}_{2} \mathrm{CO}, \mathrm{CH}_{3} \mathrm{OH}$, were measured in the radio (e.g. Drahus et al. 2008; Biver et al. 2008) and near-infrared (e.g. Bockelée-Morvan et al. 2008, Bonev et al. 2008, Böhnhardt et al. 2008) spectral regimes, suggesting a peculiar composition, different from other comets with known organic content, including two Halley-type comets. The isotopic ratios of $\mathrm{D} / \mathrm{H}$ in water as well as $\mathrm{C}^{13} / \mathrm{C}^{12}$ and $\mathrm{N}^{15} / \mathrm{N}^{14}$ in $\mathrm{CN}$ were also studied (Villanueva et al. 2009, Bockelée-Morvan et al. 2008). Moreover, an analysis of the transient arc structures visible in narrow-band images of the CN coma (Schleicher and Woodney 2007, Woodney et al. 2008) revealed the nucleus rotation period to be equal to $5.7 \mathrm{~h}$.

Perhaps the most surprising result came from radar observations of the nucleus (Harmon et al. 2008a and b). They imaged a highly elongated bilobate body, most likely a contact binary, which would be the first such case among cometary nuclei. The rotation period appeared to be $11.4 \mathrm{~h}$ (twice the value inferred from the analysis of the $\mathrm{CN}$ coma), and the similar result was reported by Lamy et al. (2008) who analysed the HST broadband photometry of the nucleus.

The ultimate goal of our campaign dedicated to 8P/Tuttle is to investigate the composition and kinematics of the molecular coma, the rotation state of the nucleus, and the 3D gas and dust ejection patterns. We also aim at explaining the dynamical connection between $\mathrm{HCN}$ and $\mathrm{CN}$ in this comet. It is widely believed that $\mathrm{HCN}$ is the only, or at least the major parent of cometary CN (Bockelée-Morvan and Crovisier 1985), although this assumption has been questioned for comet Tuttle (Bonev et al. 2008), and for some other comets (e.g. Fray et al. 2005). Thus, we have undertaken spectroscopy of HCN at millimeter-wavelengths using the Submillimeter Telescope of the Arizona Radio Observatory on Mt. Graham, along with optical imaging of the $\mathrm{CN}$ coma (supplemented by $\mathrm{C}_{2}, \mathrm{C}_{3}$ and dust bands) realized with the 2-m telescope at the Rozhen National Observatory of the Bulgarian Academy of Sciences. Preliminary results from the spectroscopic monitoring of HCN have been already presented (Drahus et al. 2008). They confirmed the rotation period of $5.7 \mathrm{~h}$ (supporting also the multiples), based on periodic repeatability of the HCN line shape. 
In this paper we focus on the early results for $\mathrm{CN}$, including: the kinematics of the coma, periodicity in the gas ejection and the problem of nucleus rotation. The ultimate results from the analysis of the connection between $\mathrm{HCN}$ and $\mathrm{CN}$ will be published elsewhere.

\section{Observations and Data Reduction}

Observations of comet 8P/Tuttle were performed during six nights in the first half of January 2008. We used the two-channel FoReRo2 focal reducer (Jockers et al. 2000) attached to the 2-m Ritchey-Chrétien-Coudé telescope at Rozhen. The effective focal ratio was F/2.8. The blue channel of the device was equipped with the Photometrics CE200ASITe CCD and the red channel used the VersArray 512B CCD. Both chips have the same pixel size of $24 \mu \mathrm{m}$ and the image scale of $0.89 \operatorname{arcsec}_{\text {pix }}{ }^{-1}$. The HB interference filters (Farnham et al. 2000) were used to separate emissions of the $\mathrm{CN}, \mathrm{C}_{3}$, and $\mathrm{C}_{2}$ radicals and dust bands. An overview of the $\mathrm{CN}$ observations, along with the geometric circumstances, is presented in Table 1. Exposures were ranging from 300 to 1,200 s. Observations of standard stars (Hamuy et al. 1992, 1994) were performed to calibrate the flux in the comet images.

Reduction of the images consisted of, among other steps, removal of the cosmic ray hits (especially important for long exposures) and subtraction of the stellar profiles elongated by non-sideral tracking. These procedures were realised using masking and filling by interpolation. Profiles of the brightest stars were masked only, because it would be difficult to avoid some bias when interpolating. Next, we stacked small groups (most frequently pairs, rarely triplets) of consecutive frames to increase the $\mathrm{S} / \mathrm{N}$ ratio. The effective exposure times of the composite $\mathrm{CN}$ images are therefore 600,900 or 1,200 s, and the time coverage (the span between the start of the first exposure of a group and the end of the last exposure) is typically $0.36 \mathrm{~h}$ (never more than $0.49 \mathrm{~h}$ ).

Next, we used a ring-masking algorithm (A'Hearn et al. 1986) and detected very weak arc structures at the sunward side. A much better result was produced after subtraction of the mean image (produced upon averaging the whole series for a given night) from the individual frames. That procedure was applied for January 4 and 9 when about $80 \%$ of the $5.7 \mathrm{~h}$ period was covered. The mean frames were obtained using the $\mathrm{K} \sigma$ algorithm, rejecting signal which was too far from the rest of a series. This approach provided us with almost structureless, smooth profiles of the mean coma. For the nights when only a couple of frames were available (January 7, 8 and 10,11), we used the mean profile from January 9. That is because the mean image from January 4 could introduce a certain bias, as this

Table 1 Statistics of the CN images and geometric circumstances

$r, \Delta$ helio- and geocentric distance of the comet, $\phi$ phase (i.e. Sun-comet-Earth) angle

\begin{tabular}{lllll}
\hline $\begin{array}{l}\text { UT Date, } \\
\text { January 2008 }\end{array}$ & $r[\mathrm{AU}]$ & $\Delta[\mathrm{AU}]$ & $\phi[\mathrm{deg}]$ & $\begin{array}{l}\text { Number of exposures } \\
\text { original/after stacking }\end{array}$ \\
\hline $4.71-4.92$ & 1.082 & 0.258 & 61.1 & $15 / 7$ \\
$7.84-7.91$ & 1.068 & 0.275 & 64.9 & $3 / 2$ \\
$8.82-8.90$ & 1.064 & 0.283 & 65.9 & $2 / 1$ \\
$9.68-9.89$ & 1.061 & 0.291 & 66.9 & $19 / 11$ \\
$10.84-10.87$ & 1.057 & 0.300 & 67.8 & $4 / 3$ \\
$11.70-11.88$ & 1.054 & 0.309 & 68.5 & $2 / 2$ \\
\hline
\end{tabular}


date is somehow isolated from the rest of the nights, and additionally covers a different part of the rotation cycle if the $11.4 \mathrm{~h}$ period is assumed. The procedure described above requires an appropriate signal normalisation and image scaling to the same geocentric distance as on January 9, which we used as a global reference (the frame scale was $187.5 \mathrm{~km} \mathrm{pix}^{-1}$ for this date). To link photometrically all the $\mathrm{CN}$ images obtained on different nights we generated the global-mean profile of the coma by averaging the mean profiles from January 4 and 9. Then we normalised the individual and the night-mean images using this global profile. By subtracting the night-mean profiles from the particular frames we ultimately produced a several-nights-long series of the differential images whose internal photometric consistency is better than $0.01 \mathrm{mag}$. Additionally, the moments of mid-exposures were converted to the nucleocentric values by subtracting the travel time of light.

It is obvious that our images of the $\mathrm{CN}$ coma contain several percents contribution produced by sunlight scattered by cometary dust particles. Instead of subtracting this extra signal using the frames obtained with the dust band filters, we followed a different approach: We checked the influence of the dust component on our differential images of $\mathrm{CN}$. Using the same subtraction procedure as previously, but this time for the dust band frames, we revealed no transient structures at all. This does not mean that dust was emitted from the nucleus without time modulation and thus did not create dust shells. Taking into account that the dust particles are a factor of a few slower than gas and that the velocity dispersion is quite substantial (mainly due to the correlation between the velocity and size of a dust particle), the dust shells overlap each other resulting in almost stationary dust coma profile. Figure 1 shows the night-mean dust coma of comet Tuttle for January 9, together with its ring-masking version. The fan like pattern at the sunward region is quite well visible. We met almost the same situation for January 4 . The stationary profile of the dust coma within each observing night and from date to date means that our differential CN images carry pure information about the molecular coma without any influence of the dust structures.
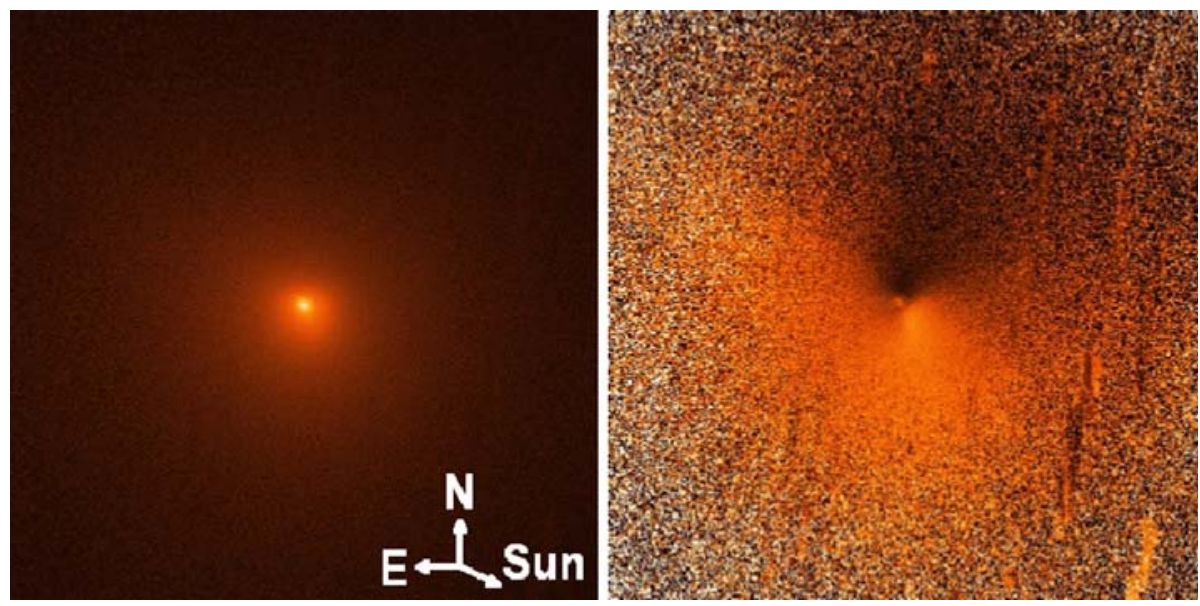

Fig. 1 Stacked image of the dust coma in the $642 \mathrm{~nm}$ band for January 11. Left panel whole night average profile, right panel its ring-masking counterpart. The size of the field of view is 5.4 arcmin or $67.7 \times 10^{3} \mathrm{~km}$ at the distance of the nucleus 


\section{CN Shells and their Kinematics}

As a result of the data reduction we have obtained a sequence of differential images, the part of which from January 9 is shown in Fig. 2 (see also Electronic Supplementary Materials for video clip Animation1).

This figure reveals shells at the contrast level of a couple of percent. They have a barely spiral shape and are much brighter towards the Sun than in the opposite direction. One can see how these $\mathrm{CN}$ structures evolve, expanding radially and diminishing as the nucleocentric distance grows, while the newly born shell appears in the vicinity of the nucleus. Similar evolution has been seen for the $\mathrm{C}_{2}$ coma, but unfortunately our sampling is not as good as for $\mathrm{CN}$ (we have only about $50 \%$ coverage of the $5.7 \mathrm{~h}$ period and only for one night).

While investigating the kinematics of the $\mathrm{CN}$ shells we intended to avoid the necessity of obtaining the actual radial distance between the shell and the position of the comet nucleus. It would not be precise enough because of a diffuse nature and a low contrast of the $\mathrm{CN}$ structures. Therefore, we determined the velocities using radial shifts of the shell between the exposures. First, the differential frames were transformed to the images parametrized by the radial coordinates, with the azimuth angle oriented along the image row and the radial distance from the nucleus arranged along the column. Next, we crosscorrelated pairs of the images, to determine the radial displacement of the shell between the two exposures. We analysed the brighter (sunward), and the weaker (anti-sunward) parts of the shells separately. The mean precision of the measured radial shifts appeared to be 0.5 pix $(0.4$ arcsec) for the first case, and 1.3 pix ( 1.2 arcsec) for the second case. Our goal was to obtain a function relating the radial position of the pattern with time, but we had only a series of two-point samples of the slope of this dependence, where each sample was produced by each pair of the cross-correlated images. Thus, we reconstructed this function by shifting the individual pairs of points along the radial distance, which made it possible to merge the samples altogether (in the least squares sense). The obtained dependence is presented in Fig. 3. The radial expansion velocity of the brighter (sunward) part was found to be equal to $0.96 \pm 0.03$ on January 4 , and $1.10 \pm 0.01 \mathrm{~km} \mathrm{~s}^{-1}$ on January 9. The weaker (anti-sunward) segment of the shell reached a generally lower velocity of $0.73 \pm 0.05$ and $0.80 \pm 0.02 \mathrm{~km} \mathrm{~s}^{-1}$ for the same dates. Unfortunately, for the other observing nights we did not collect enough images for a reliable determination of the velocities. It is worth noticing that the precision of this determination is very high and that the values measured for different dates are much closer to each other than are the velocities for the sunward and anti-sunward parts of the shells.

Although an interpretation of the outflow kinematics of the $\mathrm{CN}$ shells should take into account the projection effect, it is highly probable that for comet Tuttle the parent particles of $\mathrm{CN}$ expand into a relatively broad cone (further discussed in Sect. 5). Therefore, we expect the measured sunward expansion velocity to be roughly equal to the $\mathrm{CN}$ outflow velocity.

Our results are consistent with the $1.1 \mathrm{~km} \mathrm{~s}^{-1}$ radial expansion velocity of the $\mathrm{CN}$ sunward structures obtained by Woodney et al. (2008). These are, however, a bit higher than the expansion velocity of $\mathrm{HCN}$, which was found to be equal to $0.85 \mathrm{~km} \mathrm{~s}^{-1}$ (Biver et al. 2008) and $0.8 \mathrm{~km} \mathrm{~s}^{-1}$ (Drahus et al. 2008) from mm-waves spectroscopy of the nearnucleus region. This discrepancy cannot be explained by radial acceleration of the $\mathrm{CN}$ shells, which one might expect at first glance, based on the fact that the $\mathrm{CN}$ molecules are emitted from the parents with some excess energy (see e.g. Fray et al. 2005). In fact, our model analysis (see Sect. 4), assuming $\mathrm{HCN}$ as the only parent of $\mathrm{CN}$, shows, that the $\mathrm{CN}$ shells follow the same constant expansion velocity of $\mathrm{HCN}$, if only their emission from the 

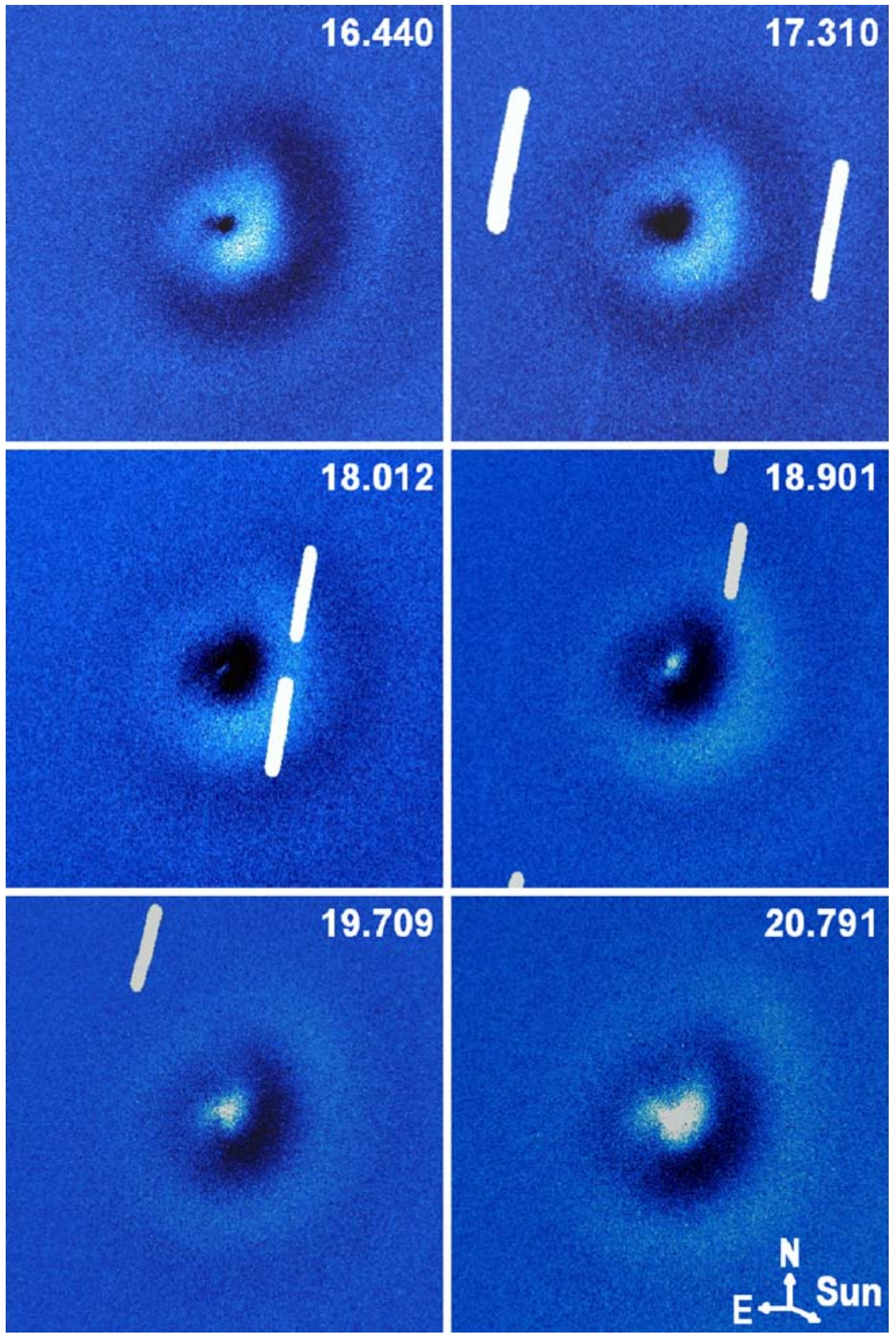

Fig. 2 Series of the $\mathrm{CN}$ images for January 9 obtained after subtraction of the mean profile. To save space only every second image is shown. The UT moments displayed in the upper-right corners have been retarded to the nucleocentric values and are given in hours. Orientation of the frames is the same as in Fig. 1, and the field of view is 5.9 arcmin or $75.2 \times 10^{3} \mathrm{~km}$ at the distance of the nucleus

parents is isotropic. Gas acceleration (stimulated by any process), the possible reason of the difference between $\mathrm{HCN}$ and $\mathrm{CN}$ velocities required to explain this discrepancy, would have to act in the transient zone between the region analysed by radio spectroscopy (20-30", 


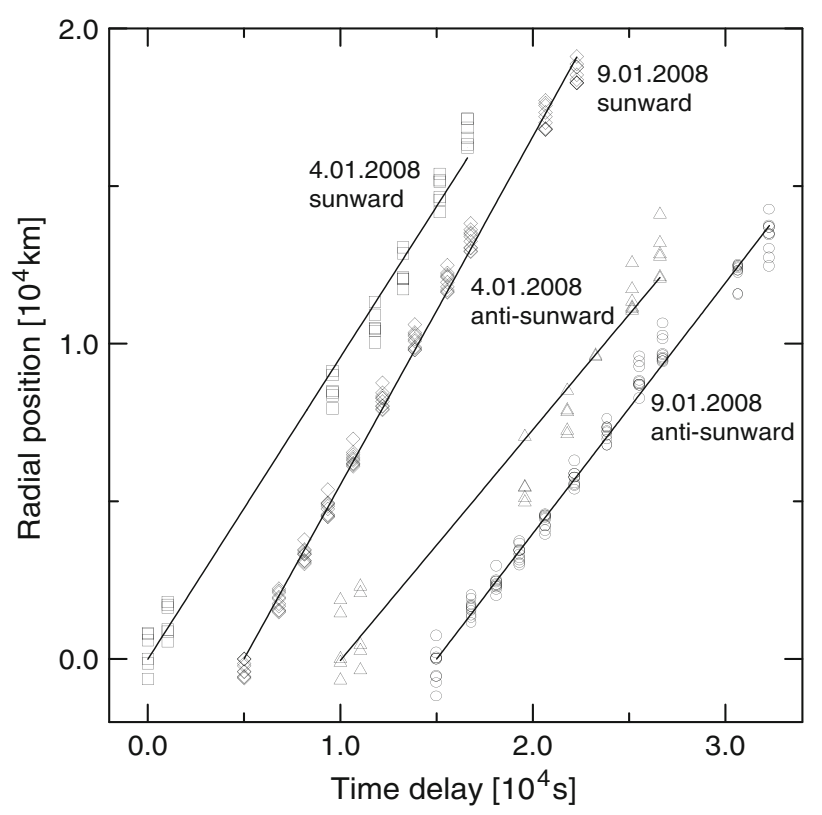

Fig. 3 Relation between the radial position and time for the $\mathrm{CN}$ shells, calculated with respect to arbitrarily chosen zero points of the position and time. The sunward part is represented by open squares (January 4) and open diamonds (January 9), and the anti-sunward section by open triangles (January 4) and open circles (January 9). The plot contains all the determined two-point samples, produced by cross-correlating pairs of the images. Since a single image was used to construct several samples, it is represented by several points at the same time instant. The segments connecting both points of each sample are not presented for transparency-we show the linear fits instead (solid lines). Error bars (not shown for clarity) are about $100 \mathrm{~km}$ for the sunward and $200 \mathrm{~km}$ for the anti-sunward arcs

what means 3,000-4,500 km from the nucleus) and sampled by our imaging (beyond 30" radius). Another likely explanation is possible underestimation of the HCN velocity, when obtained from radio spectroscopy in a standard way, as discussed by Drahus et al. (2009). This effect is expected for anisotropic gas emission from a cometary nucleus, which was clearly the case for comet Tuttle. On the other hand we should not reject the possibility that the bulk outflow velocity of $\mathrm{CN}$ behaved in a slightly different way than the outflow velocity of the $\mathrm{CN}$ shells. As was previously mentioned, the $\mathrm{CN}$ radicals forming the shells were at least one order of magnitude less abundant than the molecules forming the underlying coma. Thus, radio spectroscopy measured the bulk expansion velocity of $\mathrm{HCN}$, whereas the $\mathrm{CN}$ shells probed the kinematics of gas ejected by the active vent, where the ejection velocity could be a bit higher than for the neighbouring regions of the nucleus. Nevertheless, the $\mathrm{HCN}$ expansion velocity and the velocity of the $\mathrm{CN}$ shells were both fairly close to $1 \mathrm{~km} \mathrm{~s}^{-1}$, which is considered as canonical for comets around $1 \mathrm{AU}$ from the Sun.

\section{Periodicity in the CN Shells}

The series of the differential $\mathrm{CN}$ images gave a good opportunity to analyse the periodic behaviour of gas emission in comet Tuttle. Three almost independent approaches were used. 
First, we investigated repeatability of the $\mathrm{CN}$ structures. For this purpose we introduced a Discrepancy Measure (hereafter DM), which indicates the level of similarity between the coma patterns visible in two different images. It is defined as a mean square signal discrepancy (measured between the corresponding pixels) minus the mean square noise in both frames. The first term actually measures how similar the structures are, however, it cannot distinguish a truly structural dissimilarity from an artificial dissimilarity introduced by noise. This ambiguity is naturally removed by the second term, making the DM fairly insensitive to variations in the image quality. A series of $\mathrm{N}$ images makes it possible to calculate N(N-1)/2 Discrepancy Measures, which is equal to 325 for 26 images in our case (see Table 1). If any periodicity exists in the evolution of the $\mathrm{CN}$ shells we should observe it in a time-series of the DM points. The dependence between a DM value and a time delay between both frames behaves in some aspects like a structure function of the temporal evolution of the coma profile. We note, that this novel approach is weakly biased by small changes of the shape of the $\mathrm{CN}$ structures, which may be produced by e.g. changes of the viewing geometry. What is more, the results are completely independent of the variability in the gas expansion velocity with the nucleocentric distance. On the other hand, the method is relatively sensitive to changes of this velocity from one night to another, which seems to be the case for the two investigated dates (January 4 and 9). Test computations involving a model coma with transient arcs proved a high sensitivity of this approach. We were able to detect a periodic behaviour of the DM samples for the arc structures with a signal modulation as low as a factor 1.5 above the noise level, which was a markedly worse case than our real data. To reveal periodicity in a set of the DM values, we used the Phase Dispersion Minimization (PDM) analysis and a least squares fit of a sum of harmonics (details of both techniques can be found elsewhere, e.g. Drahus and Waniak 2006). A result of the PDM search with typical settings is presented in Fig. 4. It must be stressed that the positions of the deepest minima appeared to be barely dependent on the combination of the numbers of bins and covers. The harmonics fit provided us with very similar output when maximum one harmonic was used along with the base sinusoid. Additional harmonics produced a substantial instability of the periodogram.

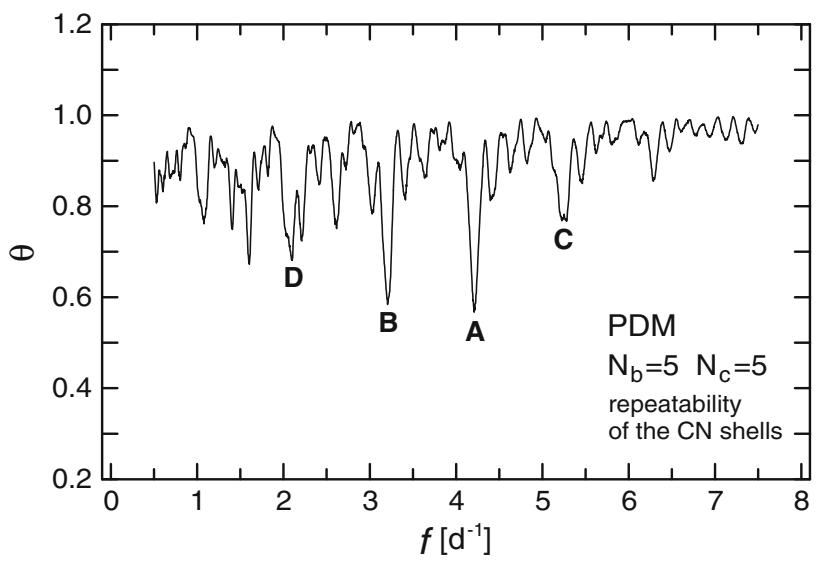

Fig. 4 Result of the PDM periodicity search for the Discrepancy Measure, presented as a dependence between the $\theta$ parameter and frequency $f$. The numbers of bins and covers were both equal to 5 . The minimum labelled by $A$ corresponds to the main period of $5.7 \mathrm{~h}$. The minima marked as $B$ and $C$ result from aliasing of the main frequency by the $1 \mathrm{~d}^{-1}$ frequency. Minimum $D$ is created by a $11.4 \mathrm{~h}$ periodicity 
The most distinct minimum visible in Fig. 4 (labelled by A) corresponds to the period of $5.70 \pm 0.07 \mathrm{~h}$ and the second and the third deepest ( $\mathrm{B}$ and $\mathrm{C}$ respectively) result from an interference between this frequency and a one-day periodicity characteristic for our observing run. Figure 5 (filled symbols) shows the DM values phased according to the period of 5.7 h. Our result is consistent with other findings (Schleicher and Woodney 2007, Woodney et al. 2008) based on the CN sunward arcs.

Another approach to investigate the periodicity in our data was to perform an aperture photometry of the central region of the $\mathrm{CN}$ coma in the differential images. This method resembles the Image Subtraction Method (ISM) used for a differential photometry of variable stars in crowded stellar fields. In our case the normalisation of the individual CN images to the common mean coma profile enables detection of the near-nucleus variability produced by gas periodically emitted by the nucleus. Our photometry in the differential images has some advantages over its original version, being substantially less sensitive to variable PSF, image scaling and orientation. On the other hand, the result of this approach may depend on the changes of the viewing geometry, the time scales of parent and daughter molecules (not so dangerous in our case), and at smaller extent, on the expansion velocity. The photometry was done in a diaphragm of 14 arcsec in radius, which was approximately the same as the half-width radius of the PSF for the HCN monitoring (Drahus et al. 2008). Fortunately, non of the stellar trails, which appeared in our images, coincided with the near-nucleus region. The photometric time-series was investigated towards periodicity with the same methods as were the DM points. The PDM periodogram, presented in Fig. 6, shows the same characteristic frequencies which can be seen in Fig. 4. Also the least squares harmonics fit provided very similar results as previously. The light

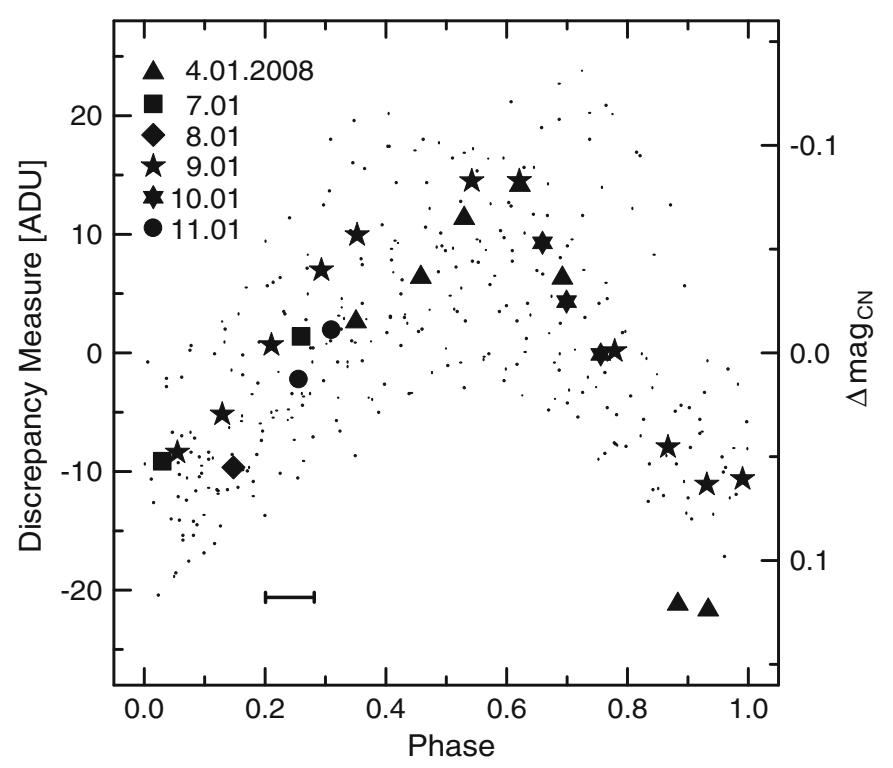

Fig. 5 Discrepancy Measures after subtraction of the mean value (dots) and aperture photometry of the central part of the coma (symbols) phased using the $5.7 \mathrm{~h}$ period. The light-curve was shifted in phase to be consistent with the DM variability curve. The segment close to the lower-left corner corresponds to a $0.49 \mathrm{~h}$ time span, which is the upper limit for the individual stacked images of $\mathrm{CN}$ 


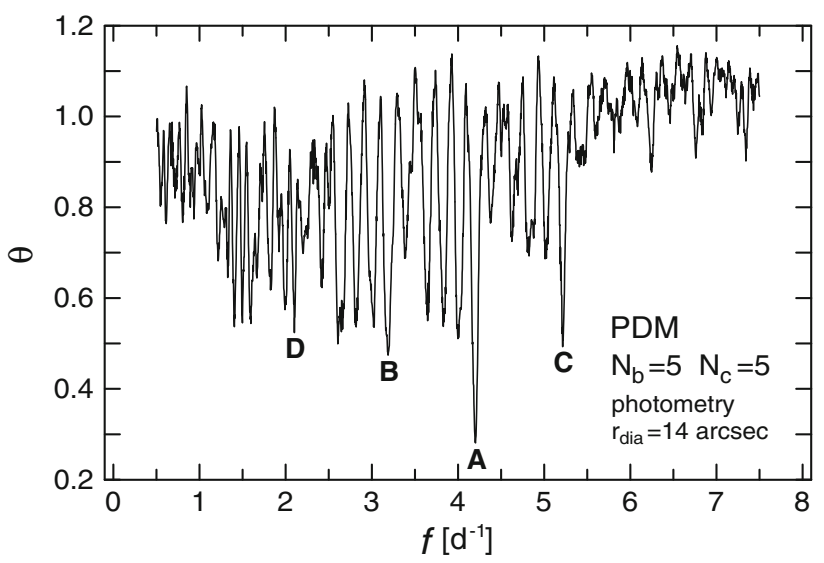

Fig. 6 Dependence between the $\theta$ parameter and frequency $f$ obtained with the PDM method implemented for the aperture photometry of the central part of the coma. The numbers of bins and covers were set equal to 5. The minima are labelled as in Fig. 4

curve phased according to the most probable period of $5.70 \mathrm{~h}$ is shown in Fig. 5 (open symbols).

The third method took into account both the kinematics of the $\mathrm{CN}$ structures and their repeatability with time. On January 4 and 9 we observed the CN shells from two consecutive generations but with only $80 \%$ coverage of the expected period. However, using the measured expansion velocities (Sect. 3) and the cross-correlation approach to determine the radial lag between the innermost shells from the two consecutive generations, it was possible to compute the time interval after which the newly created shell would reach the position of the older structure. Table 2 gives the results of such an analysis for both dates, separately for the sunward and anti-sunward parts of the shells.

The commonly appearing periodicity of $5.7 \mathrm{~h}$ is again fully supported by the results for January 9. However, for January 4 we obtained substantially longer periods, which is a puzzle to be resolved. One possibility is variation of the radial expansion velocity with the projected nucleocentric distance. Another, a substantial change of the shape of the CN envelope between the exposures. It can be also an instrumental artefact, affecting the measured radial displacement of the shells, since quality of the images and the on-comet tracking were markedly worse for this date than for January 9.

Since different authors proposed two different rotation periods for comet Tuttle, i.e. 5.7 and $11.4 \mathrm{~h}$, this ambiguity should be addressed in detail. Assuming the longer period, it becomes obvious that our data for January 4 and 9 are separated by half of the rotation cycle, and thus the observed structures were produced by two different active regions. It is visible in Fig. 5 that on January 4 the photometric points followed the general trend but the

Table 2 Periodicity in the $\mathrm{CN}$ shells obtained from the radial displacements between the arcs from the consecutive generations and using our measured velocities of the radial expansion

\begin{tabular}{lll}
\hline UT Date, January 2008 & Period (sunward; h) & Period (anti-sunward; h) \\
\hline $4.71-4.92$ & $6.20 \pm 0.03$ & $6.33 \pm 0.15$ \\
$9.68-9.89$ & $5.59 \pm 0.04$ & $5.54 \pm 0.06$ \\
\hline
\end{tabular}


last two points are markedly dispersed. We do not exclude that this is only an artefact produced by the normalisation of the images. But if not, this means that we observed either two active regions (11.4 h period) with slightly different activity or a single active vent (5.7 $\mathrm{h}$ period) which slightly evolved between January 4 and 9 . We addressed these two scenarios by comparing two groups of the differential images, one for January 4 and one for January 9, selected in such a way, that the phase delays between the frames from both samples were as close as possible to half of the $11.4 \mathrm{~h}$ rotation cycle (see Fig. 7). We conclude, that the corresponding profiles from these groups look similar to each other. The lower contrast of the anti-sunward arcs for January 4 may result from a slightly different viewing geometry and/or from an intrinsic variation of the emission pattern of the comet nucleus.

\section{Toy Model of HCN-CN Coma}

Finally we tried to interpret the results in the frame of a unique scenario of gas production by the rotating nucleus. Our only aims were to explain the transient behaviour of the profile of the $\mathrm{CN}$ coma and to test which scenario is more likely: the rotation period of $5.7 \mathrm{~h}$ and a single active region, or a twofold period and two active regions. We used quite simple Monte Carlo model to describe the $\mathrm{HCN}-\mathrm{CN}$ coma, which was a trial version of what we plan to use for the complete study of the HCN-CN connection. In our model $\mathrm{HCN}$ is the only source of CN (see Sect. 1). The HCN molecules are emitted from a single active vent or two regions separated by $180^{\circ}$ degrees in longitude. Alternatively, $\mathrm{CN}$ parents are emitted from particles (e.g. ice or $\mathrm{CHON}$ dust) forming a jet. The opening angle of the $\mathrm{HCN}$ emission cone is one of the model parameters. The activation function was equal to the cosine of the solar zenith angle. The HCN molecules move through the coma with a constant expansion velocity of $1.0 \mathrm{~km} \mathrm{~s}^{-1}$, according to our previous results (see Sect. 3), and photodissociate into $\mathrm{CN}$ at the rate of $1.3 \times 10^{-5} \mathrm{~s}^{-1}$, which is an intermediate value among all the presented in literature (see e.g. Bockelée-Morvan and Crovisier 1985; Crovisier 1994; Fray et al. 2005). Due to the excess energy, the CN radicals are ejected isotropically form the $\mathrm{HCN}$ molecules with the velocity of $1.0 \mathrm{~km} \mathrm{~s}^{-1}$ in the frame of the mass centre.

First, we have checked how much the profiles of the $\mathrm{HCN}$ and $\mathrm{CN}$ coma can differ. Figure 8 shows the $\mathrm{HCN}$ and $\mathrm{CN}$ spiral structures for the scale and viewing geometry of comet Tuttle on January 9, with the North pole directed opposite to an observer and a single point source (nucleocentric latitude of -60 degrees) sublimating $\mathrm{HCN}$ into a cone of 7 degrees opening angle. It is obvious that within one scale length (the ejection velocity divided by the photodestruction rate) from the nucleus the $\mathrm{CN}$ radical is a good tracer of $\mathrm{HCN}$. This is because photodissociation of $\mathrm{HCN}$, which spreads spiral patterns and diminishes their contrast, takes place shortly after the precursor of $\mathrm{CN}$ reaches the position of this pattern. In contrast, at larger distances, the source of $\mathrm{CN}$ is weaker (declines exponentially with time i.e. with the nucleocentric distance), and the newly created radicals become only a minor contribution to the diffuse background created by the older ones.

Using the $5.7 \mathrm{~h}$ rotation period of the nucleus and choosing different nucleocentric latitudes of the active region and different opening angles of the emission cone, we produced sequences of synthetic $\mathrm{CN}$ images covering the full rotation period. Then, we produced series of the differential images, following the same procedures as before (see Sect. 2), and compared them with the series of the observed $\mathrm{CN}$ differential frames. This led us to the conclusion that the emission of HCN took place from a small active area, or 


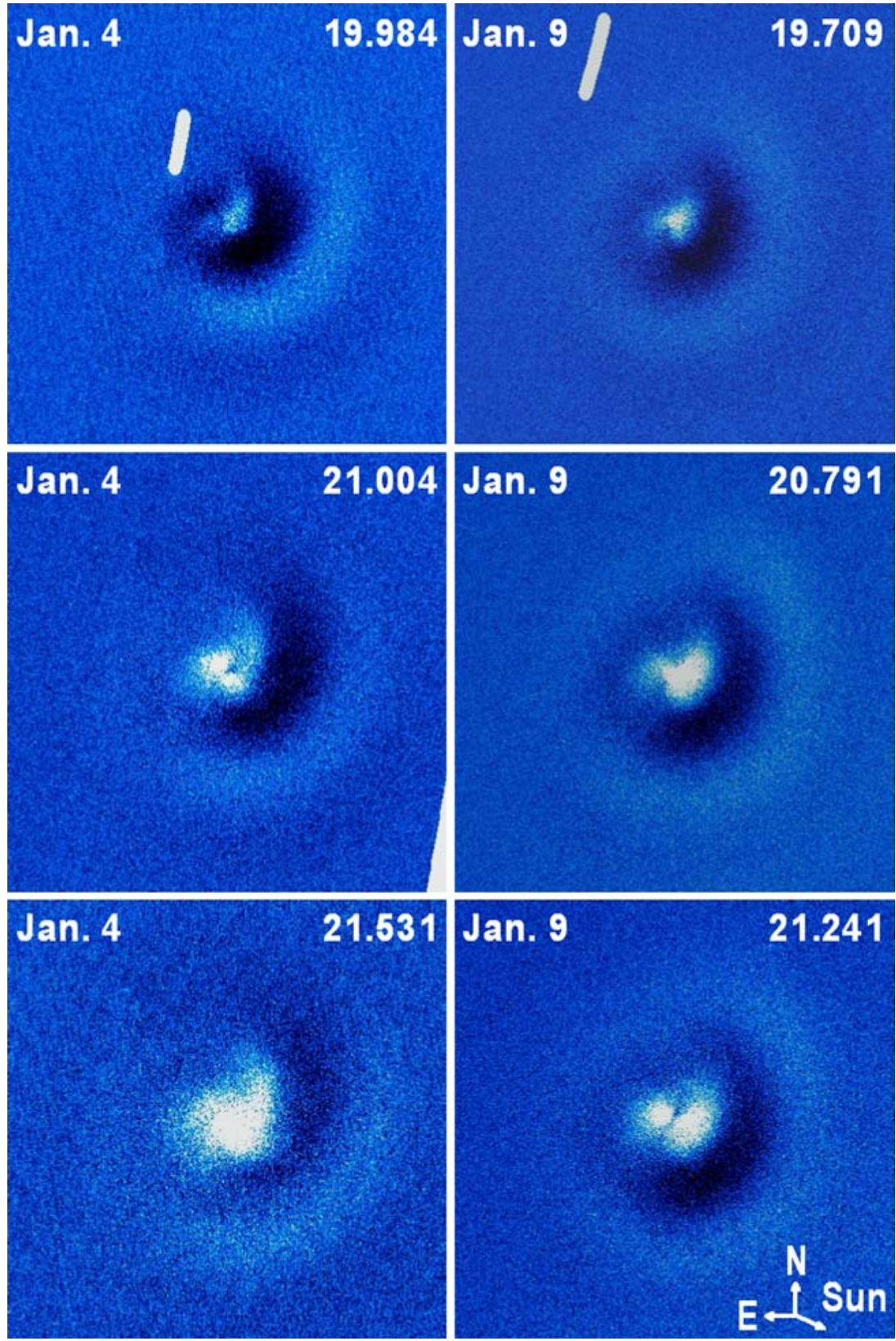

Fig. 7 Two groups (left column for January 4, right column for January 9) of the differential CN images separated in phase by about 0.5 (with a precision better than 0.01 ) if the rotation period was equal to $11.4 \mathrm{~h}$. In such a case they should have been produced by two different active regions. The UT moments shown in the upper-right corners have been retarded to the nucleocentric values and are given in hours. Orientation and scales are the same as in Fig. 1 

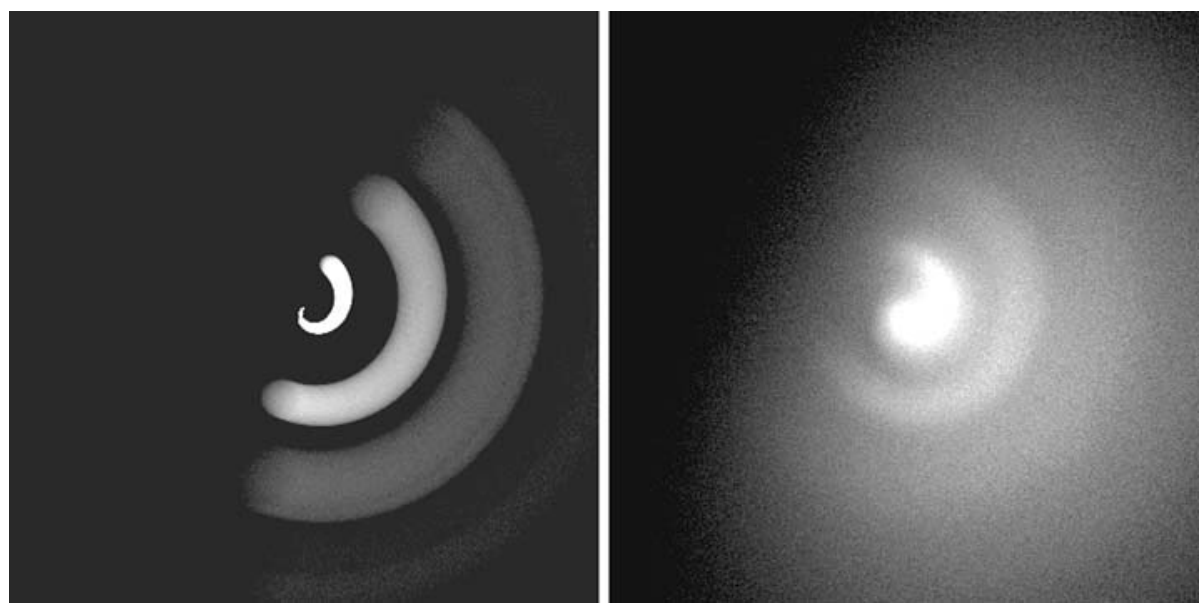

Fig. 8 Model profiles of the $\mathrm{HCN}$ coma (left panel) and the $\mathrm{CN}$ coma (right panel). Image scale and viewing geometry are the same as for comet Tuttle on January 9. The rotation axis is directed to an observer and the gas emission cone has the opening angle of 7 degrees

from a narrow dust jet created by such an area. The emission should be into a relatively broad cone (opening angle close to 70 degrees) to create the $\mathrm{CN}$ shells in good qualitative agreement with the observed pattern. This means that we need an expanding 3D structure, which is similar to a hemispherical envelope rather than to a spiral jet, since only the first scenario creates a ring pattern such as visible in our differential images. In the frame of this scenario it is quite understandable that the expansion of the sunward part of the envelope is faster (and close to the overall gas outflow velocity) than is the anti-sunward part, where the projection effect could be significant. In addition, from the analysis of the kinematics of the synthetic $\mathrm{CN}$ structures we found, that their radial projected velocity was $1.008 \pm 0.002 \mathrm{~km} \mathrm{~s}^{-1}$, very close to the assumed outflow velocity of the HCN molecules. This was the reason why we put in our model the $\mathrm{HCN}$ expansion velocity of $1.0 \mathrm{~km} \mathrm{~s}^{-1}$ which is close to the values found in Sect. 3 for the sunward part of the $\mathrm{CN}$ envelope.

Scenarios different from the one, presented above, i.e. a markedly narrower HCN emission from a small active vent or from a narrow dust jet, as well as a large active region with the size comparable to the nucleus radius emitting HCN perpendicularly to the nucleus surface, generate typical spiral structures which were not present in the differential images of the real coma. The question arises then, how it is possible to induce such a substantial divergence of a narrow jet into a relatively wide cone? This effect could be explained either by hydrodynamical broadening of the gaseous jet which carriers the HCN molecules (see e.g. Körösmezey and Gombosi 1990, Gombosi 1991) or by highly isotropic emission of HCN from ice/dust grains forming a relatively narrow jet (see e.g. Schulz 1993, Dello Russo et al. 1998).

We found the latitude of the active area of $-60^{\circ}$ and the nucleus North pole directed opposite to the Earth on January $9\left(\alpha=2.1^{\mathrm{h}}, \delta=-7^{\circ}\right.$, epoch 2000.0) as a quite good first guesses. This means that a great bulk of outgassing took place at the sub-Earth region of the nucleus, which is consistent with the blue shifted spectral lines found at the radio wavelengths (Biver et al. 2008; Drahus et al. 2008).

Next we made similar experiments with the $11.4 \mathrm{~h}$ rotation period and two properly situated identical active areas. Figure 9 presents the synthetic differential images for the 

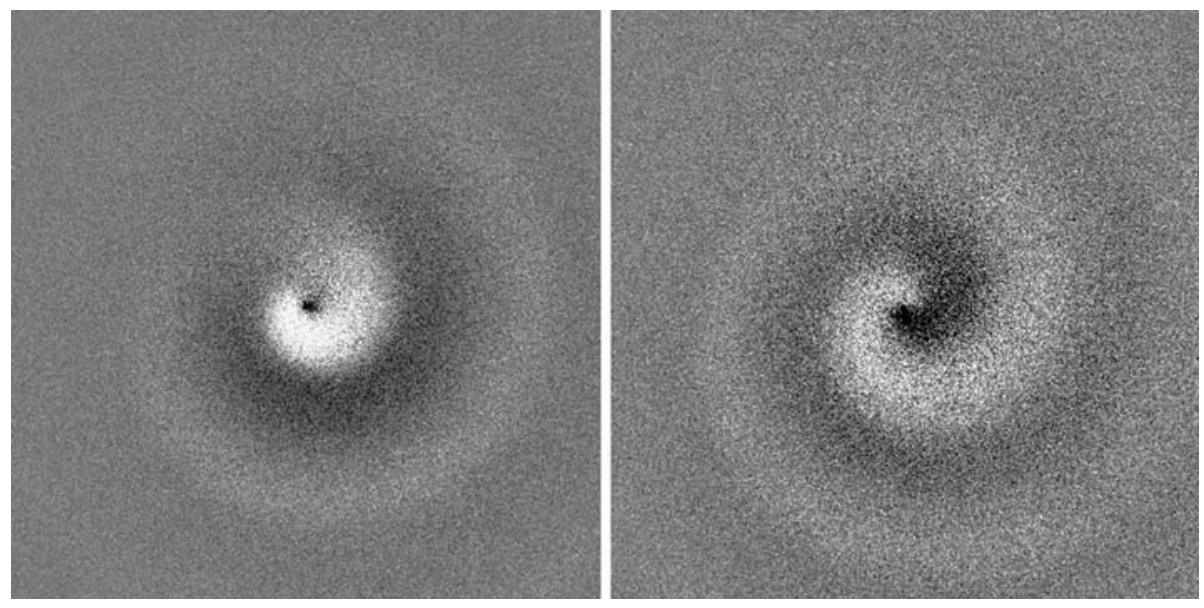

Fig. 9 Model differential images of the CN coma for January 9. One active area (left panel) or two regions (right panel) were located at the nucleocentric latitude of -60 degrees. For the right frame the separation in longitude between both active regions was 180 degrees. The rotation period was $5.7 \mathrm{~h}$ for the left panel and $11.4 \mathrm{~h}$ for the right panel. HCN was emitted from a short jet into a cone with the opening angle of 70 degrees. The North rotation pole of the nucleus is directed opposite to the Earth. Orientation and scale of the images are the same as in Fig. 1

two investigated scenarios (see also Electronic Supplementary Materials for video clip Animation 2 for $5.7 \mathrm{~h}$ and Animation 3 for $11.4 \mathrm{~h}$ periods). Both frames can be confronted with the observed differential $\mathrm{CN}$ profiles (Fig. 2). We conclude that it is much easier to reconstruct the observed pattern implementing a $5.7 \mathrm{~h}$ rotation period of the nucleus. It is simply because the angular velocity for the $11.4 \mathrm{~h}$ rotation period is too slow (comparing with the $\mathrm{HCN}$ expansion velocity) to create the observed nearly-circular $\mathrm{CN}$ envelopes, producing instead spiral structures.

\section{Conclusions}

The narrow-band $\mathrm{CN}$ images of comet Tuttle revealed almost circular envelopes arising one by one in the near nucleus region, expanding radially and then diminishing in the background of a diffuse $\mathrm{CN}$ coma. The radial velocity of their sunward motion was close to $1.0 \mathrm{~km} \mathrm{~s}^{-1}$, and the projection effect was at most very weak. We did not find any indication of the radial acceleration in the coma beyond 4,500 $\mathrm{km}$ from the nucleus. Only a couple of percent of the whole outflow of the parent molecules of $\mathrm{CN}$ underwent the modulation with time, being most probably emitted from an active vent on the rotating nucleus. The rest of the $\mathrm{CN}$ coma was created from a steady-state anisotropic source, originating predominantly from the sub-solar hemisphere of the nucleus. Taking into account the clear variability of the HCN line profiles (Drahus et al. 2008), it appears as highly probable that the source of the background $\mathrm{CN}$ was different from HCN. A potential candidate is dust, which we found to be structureless, and which was already suggested as a possible direct source of $\mathrm{CN}$ (e.g. Fray et al. 2005), but a definite answer requires further work.

Repeatability and kinematics of the $\mathrm{CN}$ shells, as well as the relative photometry of the near-nucleus region, provided us with a $5.7 \mathrm{~h}$ periodicity. This is half the value found from 
the radar observations and from the HST photometry, which both exclude this shorter rotation period. Although the $11.4 \mathrm{~h}$ cycle is also discernible in our data, it could be an artefact as the periodicity-analysis methods used by us poorly distinguish the main periods from their multiples. However, the only possibility to reconcile both solutions is to assume that the $11.4 \mathrm{~h}$ rotation period is indeed the real one, and that the nucleus had two active areas of very similar gas production rates located at similar nucleocentric latitudes and separated in longitude by 180 degrees. It is visible in Fig. 5 that a 10 degrees lapse from this longitudinal separation is sill possible. The intriguing possibility would be that each active vent is located at each vertex of the bilobate nucleus. Unfortunately, by testing both periods using our $\mathrm{HCN}-\mathrm{CN}$ model, we noticed, that it is much more difficult to obtain nearly circular envelopes for the longer one. On the other hand, we cannot exclude that a more sophisticated model of the HCN-CN coma, formally fitted to the observations, will provide us with a reasonable solution also for the rotation period of $11.4 \mathrm{~h}$. Therefore, the presented "toy model" should be considered as a starting point for a more advanced construction.

Acknowledgements The authors gratefully acknowledge the anonymous referee for many helpful comments and suggestions which helped to improve the paper.

Open Access This article is distributed under the terms of the Creative Commons Attribution Noncommercial License which permits any noncommercial use, distribution, and reproduction in any medium, provided the original author(s) and source are credited.

\section{References}

M.F. A'Hearn, S. Hoban, P.V. Birch, C. Bowers, R. Martin, D.A. Klinglesmith, Nature 324, 649 (1986) M. A’Hearn, R. Millis, D. Schleicher, D. Osip, P. Birch, Icarus 118, 223 (1995)

N. Biver, D. Lis, N. Fray, D. Bockéele-Morvan, J. Crovisier, J. Boissier, P. Colom, N. Dello-Russo, R. Moreno, N. Varvack, H. Weaver, Asteroids Comets Meteors, Abs. 8151 (2008)

D. Bockelée-Morvan, J. Crovisier, Astron. Astrophys. 151, 90 (1985)

D. Bockelée-Morvan, N. Dello Russo, E. Jehin, J. Manfroid, A. Smette, A. Cochran, D. Hutsemékers, H. Kawakita, H. Kobayashi, R. Schulz, M. Weiler, J.-M. Zucconi, C. Arpigny, N. Biver, J. Crovisier, P. Magain, H. Rauer, H. Sana, R. J. Vervack, H, Weaver, Asteroids Comets Meteors, Abs. 8190 (2008)

H. Böhnhardt, M.J. Mumma, G.L. Villanueva, M.A. DiSanti, B.P. Bonev, M. Lippi, H.U. Käufl, Astrophys. J. 683, L71 (2008)

B.P. Bonev, M.J. Mumma, Y.L. Radeva, M.A. DiSanti, E.L. Gibb, G.L. Villanueva, Astrophys. J. 680, L61 (2008)

J. Crovisier, J. Geophys. Res. 99, 3777 (1994)

N. Dello Russo, M.A. DiSanti, M.J. Mumma, K. Magee-Sauer, T.W. Rettig, Icarus 135, 377 (1998)

M. Drahus, W. Waniak, Icarus 185, 544 (2006)

M. Drahus, C. Jarchow, P. Hartogh, W. Waniak, T. Bonev, G. Borisov, K. Czart, M. Küppers, Asteroids Comets Meteors, Abs. 8334 (2008)

M. Drahus, M. Küppers, C. Jarchow, L. Paganini, P. Hartogh, G. L. Villanueva, Astron. Astrophys. (2009, accepted)

T.L. Farnham, D.G. Schleicher, M.F. A’Hearn, Icarus 147, 180 (2000)

N. Fray, Y. Bénilan, H. Cottin, M.-C. Gazeau, J. Crovisier, Planet. Space Sci. 53, 1243 (2005)

T. I. Gombosi, Comets in the Post-Halley Era, ed. by R. L. Newburn Jr. (Kluwer Academic Publishers, Dordrecht, The Netherlands, 1991), pp. 991-1001

M. Hamuy, A.R. Walker, N.B. Suntzeff, P. Gigoux, S.R. Heathcote, M.M. Phillips, Publ. Astron. Soc. Pacific 104, 533 (1992)

M. Hamuy, N.B. Suntzeff, S.R. Heathcote, A.R. Walker, P. Gigoux, M.M. Phillips, Publ. Astron. Soc. Pacific 106, 566 (1994)

J. K. Harmon, M. C. Nolan, E. S. Howell, J. D. Giorgini, IAU Circ. 8909, ed. D.W.E. Green (2008a) 
J. K. Harmon, M. C. Nolan, E. S. Howell, J. D. Giorgini, Asteroids Comets Meteors, Abs. 8025 (2008b) K. Jockers, T. Credner, T. Bonev, V. N. Kiselev, P. Korsun, I. Kulyk, V. Roshenbush, A. Andrienko, N.

Karpov, A. Sergeev, V. Tarady, Kinematika i Fizyka Niebesnykh Tel, Suppl. 3, 13 (2000)

A. Körösmezey, T.I. Gombosi, Icarus 84, 118 (1990)

P. L. Lamy, I. Toth, L. Jorda, H. A. Weaver, O. Groussin, M. F. A'Hearn, American Astron. Soc. DPS meeting \#40, \#5.02 (2008)

H.F. Levison, ASP Conf. Ser. 107, 173 (1996)

J. Licandro, G. Tancredi, M. Lindgren, H. Rickman, R.G. Hutton, Icarus 147, 161 (2000)

D. G. Schleicher, L. Woodney. IAU Circ. 8906, ed. D.W.E. Green (2007)

C. Snodgrass, S.C. Lowry, A. Fitzsimmons, MNRAS 385, 737 (2008)

R. Schulz, Astron. Astrophys. 268, 319 (1993)

G.L. Villanueva, M.J. Mumma, B.P. Bonev, M.A. Di Santi, E.L. Gibb, H. Böhnhardt, M. Lippi, Astrophys. J. 690, L5 (2009)

L. M. Woodney, D. G. Schleicher, A. N. Bair. Asteroids Comets Meteors, Abs. 8316 (2008) 